\title{
SOME REFLECTIONS ON THE TRICHOMONAS VAGINALIS*†
}

\author{
BY \\ NEVILLE MASCALL \\ From the Endell Street Clinic, St. Peter's, St. Paul's, and St. Phillip's Hospitals, London
}

Eighteen years have elapsed since Mr. A. J. King, Dr. Orpwood Price, and I produced our paper on Trichomonas vaginalis (King and others, 1936), and $I$ think $I$ am right in suggesting that it then aroused considerable interest in the problem of trichomonad infestation. The protozoan was regarded merely as a harmless saprophyte, as had happened during much of the time since Donné (1836) first drew attention to its presence in the vaginal exudate of women suffering from vaginitis, and the whole subject was, in general, overlooked.

Looking back, I have been wondering what advances, if any, have since been made in our knowledge of the life cycle of this trichomonad, its infestation of the vagina, and the treatment of the resultant vaginitis. On first thoughts I was inclined to say none, in spite of the considerable amount of literature that has been published; but the papers of Lanceley (1954), McEntegart (1954), and Feinberg (1953) encourage me to say that there has been slight progress on the laboratory side.

It is my intention in this short paper to put forward certain ideas on the subject. I know very well that some of you will not agree with them, but in that case it is up to you to suggest alternatives. Many are my own views based on observations, experience, and reading during the last 20 years, in which my interest has been ever alive; most of them are supported by the findings of other workers.

I do not propose to deal in detail with the symptoms and signs of trichomonad infestation of the vagina, but I consider irritation to be the most marked and universal symptom, though I am led to believe from questioning patients that it is not so intense as that accompanying the monilial infection due to Candida albicans.

Let us, at the outset, admit that we are still very much in the dark regarding trichomonad infestation. Here, for instance, are a few of the problems which

* Received for publication, May 26, 1954

†Presidential Address read to the M.S.S.V.D. on April 30, 1954 beset us: How does the Trichomonas vaginalis enter the human host? Is it a purely venereal infection? Why are females apparently infested so much more often than males ? Is the organism a true pathogen? Why is the infestation in many cases so resistant to treatment ?

We are still ignorant of this common condition which probably causes more misery and discomfort than anything similar known to woman, and there has been far too little organized research.

ORIGIN OF THE INFESTATION.-Up to the present time, so far as I am aware, no definite conclusions have been reached as to how the trichomonads gain access to the body. I should like first to consider the venereal origin, as there is no doubt that in some cases the trichomonads are transmitted venereally. If a careful history is taken from infested patients one is struck by the frequency with which the onset of characteristic symptoms follows sexual intercourse. In some cases the onset is within 3 to 10 days thus closely resembling the incubation period of a typical gonococcal infection. The connexion here is more than a mere coincidence. Coital infection may arise from direct inoculation with infected semen or by contact with infected material harboured behind the prepuce. This is a favourite lurking site for the trichomonads and on two occasions I have seen very severe ulceration of the glans and coronal sulcus from this cause. Sexual intercourse may stir up a dormant infection by the outpouring of the cervical secretion together with that of the accessory sexual glands (Bartholin's gland, etc.). Local trauma during intercourse may also awaken a latent focus of infection.

I have recently surveyed 600 patients with vaginal discharges of whom 274 (45.6 per cent.) were suffering from trichomonas vaginitis, 260 (43.3 per cent.) from gonococcal infection, and 84 (14 per cent.) from monilial infection. Of the 274 who were infested with trichomonads, 132 (48.1 per cent.) 
were single women, 120 (43.7 per cent.) were married, and there were 22 ( 8 per cent.) others, including widows and those separated or divorced. All admitted sexual intercourse. Thus it is seen that trichomonas vaginitis is definitely not a disease of the married alone, but rather of the sexually active, there being an excess of 4.4 per cent. infections amongst the unmarried. Donald (1952) also stressed this point in reviewing his series in which, however, there were twelve $(5.4$ per cent.) virgins, as he states, " in both the letter and the spirit".

There were 22 pregnant patients among my 600 , and sixteen of these $(72.7$ per cent.) were trichomonas positive. Furthermore, the 274 trichomonaspositive patients divided into age groups at which the infestation, from the onset of symptoms, appeared to occur gave the result set out in Table $I$. I think that these figures again simply indicate that the majority of infestations occur during the period of greatest sexual and ovarian activity, as would naturally be expected.

TABLE I

AGE AT ONSET OF SYMPTOMS

\begin{tabular}{c|c|c}
\hline Age Group & No. of Cases & Percentage \\
\hline $15-19$ & 48 & $13 \cdot 9$ \\
$20-24$ & 102 & $37 \cdot 2$ \\
$25-29$ & 60 & $21 \cdot 8$ \\
$30-34$ & 26 & $9 \cdot 4$ \\
$35-39$ & 20 & $7 \cdot 2$ \\
$40-44$ & 12 & $4 \cdot 4$ \\
$45-50$ & 6 & $2 \cdot 2$ \\
\hline
\end{tabular}

Venereal transmission may explain part of the picture, but I am not convinced that it is the whole answer. There is too great a disparity between the incidence of the infestation in the male and in the female. In the most recent studies of the male trichomonas infestation the incidence is estimated as varying between 5 and 20 per cent., though in three of my series, each of 100 cases, the highest incidence was 2 per cent. The highest incidence seems to occur in the Negro population of America. In my recent review of 600 female patients, the incidence, as already stated, was 45.6 per cent. Furthermore, Berstine and Rakoff (1953) in a series of sixty husbands of infested women found only four to be affected, although all admitted to frequent intercourse with their wives without protection. In a series of 150 contacts whom I examined, only six infested males were found. Surprisingly, however, Whittington (1951a), examining semen by cultures, as well as by wet preparations, of the husbands of 26 women suffering from trichomonas vaginitis found seven to be positive, thus giving the high incidence of 27 per cent. Only one of the contacts had subjective symptoms.
In a control group of ten husbands of normal women no trichomonads were found.

Digressing for a moment, I should like to stress here that I do not agree with the view held by some of our American colleagues and others that trichomonas infestation in the male is a trivial affair and that it easily dies away of its own accord. Sometimes the infestation does rapidly respond to treatment, but I have on occasions found it very persistent, relapsing, and most difficult to eradicate. It is of interest to note that, at one time, when it occurred in bulls, it was regarded as incurable and the beasts were slaughtered.

By what other means can the infection be spread? At one time the water in swimming baths was suspected. This probably arose from the fact that it was observed that Trichomonas intestinalis and Trichomonas buccalis could live in the water of stagnant pools, but it is my experience that Trichomonas vaginalis does not like the chlorinated water used in swimming baths today. Towels, clothing, and lavatory seats have all in turn been blamed, and it is my opinion that the infestation may occasionally be transmitted directly by improperly cleansed and sterilized communal douche nozzles.

I should like to put forward a theory, or rather lend support to a theory, as I feel sure it must have been advanced before, that the trichomonads enter the body by ingestion, the material becoming infected through the medium of flies and animals. Dogs and cats are notoriously liable to infestation with trichomonads, and it has been shown by Dobell (1934) that a single species of trichomonad can live in different hosts, for example monkeys and men, also in different situations, such as the intestine and the vagina, and that there may be no symptoms. Hegner (1925) stated that the trichomonad can survive passage through the ordinary house fly, and it has also been demonstrated adhering to the feet of flies. It is also interesting to note from the epidemiological standpoint that all strains of trichomonads will live for a time in milk though they do not give evidence of multiplying in this medium. I have kept them by this means and have observed them alive after $48 \mathrm{hrs}$ when kept in the incubator at $37^{\circ} \mathrm{C}$. though they had disappeared at $56 \mathrm{hrs}$. Whittington (1951b) has also demonstrated that they may survive in tap water.

The obvious criticism of this theory lies in the fact that the male does not become infested to the same extent as the female. We have already seen from the incidence that the male is a poor recipient of trichomonads, but this I believe to be due to some difference in the hormonic response of the 
sexes. That a certain amount of oestrogenic activity is required for the trichomonads to reach full maturity is suggested by the following facts :

(1) Trichomonas vaginitis is practically unknown in children; it is not seen before the approach of puberty.

(2) It is never seen after the menopause has been firmly established.

(3) Some observers have found that a better growth of trichomonads is obtained when their culture medium is reinforced with oestrogenic hormones.

(4) The infestation is very common and often reaches its height during pregnancy, a time when there is an exuberance of oestrogenic hormones. This is probably due to the fact that the oestrogens produce a deposit of glycogen in the vaginal mucosa, thus providing carbohydrates which the trichomonads are able to utilize. For this reason oestrogens should not, if avoidable, be administered during treatment of an infestation.

With the object of ascertaining whether the time of onset of ovarian activity bore any relationship to infestation with Trichomonas vaginalis, the time of the menarche was ascertained from 532 patients (Table II).

TABLE II

AGE AT MENARCHE

\begin{tabular}{c|c|c}
\hline Age (yrs.) & No. of Cases & T. vag. \\
\hline 9 & 2 & - \\
10 & 12 & 8 \\
11 & 30 & 20 \\
12 & 82 & 38 \\
13 & 130 & 72 \\
14 & 132 & 56 \\
15 & 82 & 30 \\
16 & 28 & 12 \\
17 & 18 & 10 \\
18 & 12 & 4 \\
19 & 2 & - \\
20 & 2 & - \\
\hline
\end{tabular}

These statistics are not very informative and simply indicate that, as would be expected, the majority of infestations occur in women in whom the menarche occurs at about the age of 13 years. May it not be possible then that the female is the incubator and reservoir of the trichomonads and that they only develop to full maturity under certain hormonic stimulation? Here I disagree with Feo (1944), who states, in discussing the high incidence of infestation in his male series (Negro 16.46 per cent., white 12.04 per cent.), that

the male is not only responsible for reinfection of women but is the principal agent of transmission.

The differing reactions of the sexes to certain infections is well brought out in the study of syphilis, a disease with which most of us are quite familiar. Syphilis in the female takes a much milder course than in the male and this is especially so during pregnancy. Why this happens we do not know.* Though we are ignorant of what factor or factors are at work, we do know that this suppression definitely takes place and that it seems to exert its greatest influence during pregnancy. May it not, therefore, be possible that $X$ a suppressive factor for Trichomonas vaginalis operates in the male ? Personally I consider this to be a possibility and that the factor may be hormonic.

Returning to the ingestion theory, I should like to suggest that the trichomonads develop in one of three sites, the mouth, the intestine, or the vagina. In our original paper (King and others, 1936) it was suggested that :

in man the parasites isolated from various regions such as the mouth, vagina, bladder, prostate, and intestines, are specific for each of these localities, but the evidence of this is not convincing.

I am cognizant of the publications of Wenrich (1944), Liston and Liston (1939) and other workers on the comparative morphological characteristics of the trichomonads but I still do not consider their arguments convincing; I agree with Wenyon (1926) when he writes that :

it seems quite possible that the three species of $\overrightarrow{0}$ human trichomonads belong to one species and thaf on the differences which occur are due to variations in nutrition.

He goes on to state that he has studied the trichomonads of the mouth, vagina, and intestine and that the oral and intestinal forms resemble one another so closely that it is impossible to differentiate them, and that this is also true of the smaller forms which occur in the vagina :

The larger vaginal flagellates are probably overgrowth forms.

I also agree with Lynch (1922) when he states that :

it is impossible thus far to differentiate with certainty the trichomonads from the intestine, vagina, and mouth,

and also with Andrews (1929), who believed that the type of trichomonad found in the vagina could change into the type found in the intestines of men. I was also interested to hear McEntegart (1954) suggest that there might be some connexion between the intestinal and vaginal trichomonads, and that it has been stated that their serological reactions are identical.

The specific theory is based mainly on the number of flagella possessed by the protozoan, the length

* Burke (1940) had his lipoidal theory, but this was by no means generally accepted. 
of the undulating membrane, and the size of the whole organism. However, it has to be admitted that it is extremely difficult to count flagella in a wet specimen with any degree of accuracy, and on this point it is therefore impossible to be dogmatic. It is only possible simply to say that in any given specimen the trichomonad with a certain number of flagella predominated. In the same microscopic field taken from the vagina, I have observed trichomonads with three, four, and five flagella. In stained specimens it is not uncommon to see flagella detached, having apparently become so during the fixing of the specimen : evidence from such sources is therefore unreliable. It is also stated that the Trichomonas vaginalis has an undulating membrane extending along two-thirds of its body, thus differing from Trichomonas intestinalis which is said to have an undulating membrane along its entire length. I have observed, however, that just prior to the encystment of Trichomonas vaginalis the undulating membrane seems sometimes completely to surround the body of the trichomonad which it appears to roll into a ball. Also the undulating membrane of Trichomonas vaginalis may extend the whole length of the body. This is especially so in the smaller forms found in the vagina, and occurs at the time when the protozoan squeezes off posteriorly a blob of protoplasm, a process called by the Americans "autotomy". It would therefore appear that the undulating membrane is capable of considerable variation under different conditions. It is my opinion that the trichomonads reach their greatest size in the vagina because there the food is plentiful, the vaginal epithelium being rich in glycogen which makes carbohydrate readily available. I consider the number of flagella and the length of the undulating membrane both to be environmental factors, the former depending on the availability of food. Furthermore it is possible to inoculate the vagina with flagellates from other sites. Karnaky (1934) inoculated eight women, who were known to be free from infestation with Trichomonas vaginalis, with Trichomonas intestinalis. Of these, three acquired infection and the intestinal trichomonads turned over to the vaginal type in 4 to 5 days. I have succeeded, after several failures, in keeping Trichomonas buccalis alive in the vagina for 7 days, though I must admit that by that time they were not very healthy and were easily eradicated. Stabler and Feo (1942) also inoculated Trichomonas buccalis into the vagina, but again only succeeded in obtaining transient infection. I have had no experience with the intestinal type.
It has been suggested, from the fact that division of the trichomonad is rarely seen in wet specimens or in vaginal smears stained to show nuclear division, that the reproduction of the protozoan occurs somewhere other than in the vagina. Might this not be in the bowel?

Trichomoniasis and Menstruation.-No one who has studied the Trichomonas vaginalis carefully can have failed to observe the effect of menstruation upon the protozoan. It then appears to be intensely active and there tends to be a preponderance of the smaller vaginal type, the one that most nearly approaches Trichomonas intestinalis. This leads one to suspect that active reproduction or division may have been taking place. It is also a well-recorded fact that " relapse" or "flare-up" of the inflammatory process is most common during or just after menstruation, and this is a strong argument in favour of continuing treatment of the infested vagina during the menstrual period.

Digressing for a moment, let us consider how the trichomonads may reach the vagina other than venereally, thus giving rise to re-infestation or relapse. I think there are three possible ways :

(1) Through the Blood Stream.-The presence of trichomonads in venous blood drawn by venepuncture was demonstrated by Wagner and Hees (1935). I think, however, that I am right in stating that this finding has not been confirmed by other workers.

(2) Through the Urine.-I have found Trichomonas vaginalis in centrifuged specimens of urine taken from asymptomatic cases (by that I mean asymptomatic as far as the urinary system was concerned). It is well known that cystitis may be set up by Trichomonas vaginalis, and even infection of the kidney has been reported.

(3) From the Rectum.-This was suggested in our original paper as being due to the prevalent habit amongst women of wiping the anus from behind forwards.

I still do not rule out the possibility that transference from the rectum to the vagina may take place by means of a sanitary towel or pad, although this was apparently disproved by Swift (1937) who failed to demonstrate trichomonads thereon. It was with this in mind that I decided to delve into the menstrual histories of patients under investigation. As is well known, there are two types of menstrual hygiene in vogue today, namely the sanitary towel or pad and the intravaginal tampon of the Tampax type. Of the 600 patients under review a reliable menstrual history was obtained from 580. It was found that the ordinary pad or 
towel was worn by $362(60.3$ per cent.) and the intravaginal tampon by 210 ( 35.0 per cent.). Other types, such as cotton wool plugs, were worn by eight (1.3 per cent.) patients. Of those wearing the ordinary type of towel, $156(40.3$ per cent.) were infested with Trichomonas vaginalis as against 130 (61.4 per cent.) who wore the intravaginal tampon. Thus $21 \cdot 1$ per cent. more patients utilizing the tampons were infested. This, in my opinion, is due to various factors which include :

(i) trauma of the vaginal epithelium during insertion or removal of the tampon;

(ii) incomplete removal of the tampon. (Not infrequently one has to remove remnants of a tampon during routine testing and patients even forget them altogether until they report for examination for a discharge.)

(iii) infrequent changing, which probably leads to improper absorption with incomplete drainage and the retention of blood with the products of menstruation in the vaginal tract, menstrual blood being an excellent medium for the growth of Trichomonas vaginalis.

It is interesting to note that similar investigations in monilial (Candida albicans) and gonococcal infections in this series showed that there was always a preponderance of infection where vaginal tampons were used. Thus, of the patients monilially infected, 10.4 per cent. wore the ordinary type of towel, while 11.4 per cent. used the intravaginal tampon. Of those suffering from gonorrhoea, 38.6 per cent. wore the ordinary type, and 44.7 per cent. the tampon.

I think it also interesting to note here that in the 600 patients the vaginal flora was as follows :

$\begin{array}{ccc}\text { Grade } & \text { No. } & \text { Per cent. } \\ \text { I } & 142 & 23 \cdot 6 \\ \text { II } & 104 & 17 \cdot 3 \\ \text { III } & 354 & 59 \cdot 0\end{array}$

When infestation with Trichomonas vaginalis occurred the flora was :

$\begin{array}{ccc}\text { Grade } & \text { No. } & \text { Per cent. } \\ \text { I } & 28 & 10 \cdot 5 \\ \text { II } & 48 & 13 \cdot 9 \\ \text { III } & 198 & 72 \cdot 2\end{array}$

The Döderlein classification is used in the above grading of the vaginal flora :

Grade I.-Döderlein's bacilli (Lactobacillus vaginalis) alone.

Grade II.-Döderlein's bacilli with one or several other types of organisms.

Grade III.-Döderlein's bacilli absent. Various other bacteria compose the vaginal flora.

These figures show that when the vagina becomes infested by Trichomonas vaginalis there is a marked deterioration in the vaginal flora. However, 10.5 per cent. of infestations occur in women with what might be termed a normal healthy vagina. This fact undoubtedly caused the trichomonad to be regarded for many years as a harmless saprophyte that only turned into a pathogen when some damage had been caused in the vaginal epithelium by other invaders such as the streptococcus or gonococcus. A non-haemolytic streptococcus was believed by Hesseltine (1933) to be the main aetiological factor in causing the vaginitis which occurs when infestation takes place. I think, however, that the experimental work of Hogue (1944) on the effect of the Trichomonas vaginalis on tissue culture cells and the inoculation experiments of Trussell and Plass (1940) have gone far to prove that it is a true pathogen in its own right.

Combined recognized vaginal infection occurred as follows in the present series :

Infection No. Per cent. Trichomonas vaginalis and gonococci $86 \quad 31.0$ $\begin{array}{lrr}\text { Trichomonas vaginalis and monilia } & 26 & 9 \cdot 4 \\ \text { Trichomonas, monilia, and gonococci } & 8 & 2 \cdot 1\end{array}$

The 13.9 per cent. with Grade II flora could be regarded as moderately healthy, but the majority $(72.2$ per cent.) of the vaginas were definitely pathological.

Trichomonas vaginalis can be found in vaginasc with a wide range of $p \mathrm{H}$. I have found the $p \mathrm{H}$ as? low as 4 and as high as 8 (Table III). I think it is

TABLE III pH OF VAGINA

\begin{tabular}{c|c|c}
\hline$p H$ & No. of Cases & Percentage \\
\hline 4.0 & 2 & 0.7 \\
4.5 & 2 & 0.7 \\
5.0 & 25 & 9.4 \\
5.5 & 122 & 44.5 \\
6.0 & 46 & 16.8 \\
6.5 & 43 & 15.6 \\
7.0 & 25 & 9.4 \\
7.5 & 6 & 2.1 \\
8.0 & 3 & 1.0 \\
\hline
\end{tabular}

of interest to observe that with a $p \mathrm{H}$ of 4 or 8 the trichomonads appeared to be inactive, and that at 4.5 and 7.5 the majority were inactive and the remainder very sluggish.

Johnson (1942) has pointed out that there is a relationship between the size of the Trichomonas vaginalis and the reaction of the environment, the smaller types, akin to Trichomonas buccalis, being predominant when the acidity favours rapid multiplication, around the $p \mathrm{H} 5.5$ to 6.5 group. The large trichomonads, which occur when conditions are not so favourable, may be either about to divide or may be slowly degenerating. The vaginal dis- 
charge which occurs in cases of Trichomonas vaginalis infestation, although acid, is less so than normal ( $p \mathrm{H} 4.0$ to 4.5). As Table III shows, therefore, Trichomonas vaginalis practically never lives in a perfectly healthy vagina, if such a thing there be. .

TREATMENT.-As we are all very well aware the treatment for Trichomonas vaginalis infestation is still most unsatisfactory, there being as yet no known specific. Trussell (1947), in his monograph on trichomonas vaginitis, which runs to some 218 pages, devotes 70 of them to various methods of treatment, thus suggesting that the position is still pretty chaotic. Not infrequently I am approached by representatives of chemical manufacturing firms with requests to try out clinically their latest product, and at the same time they sometimes drop a tactful hint about writing up the results in the medical press. My reply is always that I will do so if their preparation proves superior to those I am already using. The number $I$ have tried is legion (I do not propose enumerating them here), but the amount of writing up I have done is practically nil. I am convinced from my experience that what suits one patient may very well not suit another ; our only hope of finding a permanent cure lies in the production of some preparation which will attack the protozoan through the blood stream, and to this end I have tried out most of the anti-malarial drugs, but with no success. The various chemical compounds now at our disposal undoubtedly cure some sufferers permanently and many more for varying periods of time, but for the majority, alas, the rule is relapse. In the series under review, 88 patients (32.1 per cent.) relapsed or became re-infested (who is to say which ?) and many defaulted. The fate of these is unknown but probably they wander from clinic to clinic hoping that some day somebody will strike on a preparation to cure them at last. In the present state of our knowledge there is no doubt that once a woman becomes infested her susceptibility to re-infestation or to exacerbations of the same infestation remains with her until some variable length of time after the menopause.

The antibiotics aureomycin and terramycin, for which some good results have been claimed in America, have in my hands proved most disappointing. With them there appears to be a considerable risk of causing a severe monilial infection, or of substituting this for the trichomonas infestation. This can occur even when the antibiotics are buffered with methyl and propyl paraben as suggested by Greenblatt and Barfield (1951) ; indeed the conditions they produce in the vagina seem ideal for the growth of Candida albicans.
At an Ehrlich Commemoration Meeting held recently in Germany, however, Professor Seigo Hosoya (1954), of the Institute of Infectious Diseases in Tokyo, described results obtained from using "Trichomycin", an antibiotic isolated from streptomyces. It was stated to be active both in vivo and in vitro against protozoa such as Trichomonas vaginalis, Treponema pallidum, and Entamoeba histolytica, as well as against several types of moulds. It was said to prevent the growth of trichophyton and Candida albicans, and to be therefore the only antibiotic effective against mycotic infections. I have had no experience of this preparation, but let us hope that it lives up to its reputation and that supplies may soon be available here.

\section{CONCLUSIONS}

In searching for a resolution of many of our difficulties in infestation of the vagina by the trichomonad (these are mainly relapse and resistance to treatment), I am led to the belief that somewhere in its life cycle there is a spore or encystment stage. The trichomonads are widely dispersed throughout the animal kingdom and spore formation has been observed in some of them. So far I have no definite proof of encystment taking place, but similar views have been expressed by many workers. Siebenthal (1945) writes of an encysted form which he says is present particularly in patients who are resistant to treatment. I think also that Donald (1952) was inclined to this theory when he wrote :

One is encouraged to believe in the existence of an encysted form which can remain dormant until conditions are more favourable. So far only pseudocysts have been found which cannot be cultured back into activity.

Lydon (1945) described a resting phase in specimens from the male urethra as cells with a "hard" limiting membrane when viewed by the dark-field method. He had difficulty in distinguishing these structures from medium-sized epithelial cells. Wenyon (1926) definitely stated that encystment occurred. Fukushima (1954) kept vaginal trichomonads in an icebox and was able to culture the organisms after 3 days' chilling. He attributed this resistance to encystment. Zinser (1941), from his studies of vital stained and fixed smear-preparations of vaginal discharge, concluded that vegetative forms of Trichomonas vaginalis develop from cysts, later become amoeboid, and finally become encysted again. Schmid and Kamniker (1926) failed to demonstrate cysts in unstained discharge, but claimed that by using Gram's stain they could detect cysts which were dark with a pale nucleus, though 
the flagella, and sometimes the undulating membrane, were missing. Barlow (1916), Hantke (1921), Balkow (1935), and Bensen (1910), also described cyst formation.

I was hoping that Dr. J. G. Feinberg would be able to reactivate the dried Trichomonas which he brought in a bottle to our last meeting in the same way as I believe some bacteriologists are able to reactivate certain frozen bacteria, but he tells me that his attempt has not been successful (Feinberg, 1953). Further improvement in cultural methods may, however, make this possible in the future. I have recently been observing the dying, or supposedly dying, Trichomonas vaginalis in culture, and have observed how the protozoan becomes practically circular in shape, how the flagella, which finally disappear, tend to wrap themselves round the protoplasm, which by this time has become granular, to form a type of cyst similar to that described in the male by Lydon (1945). I have never seen them disintegrate entirely, and I do know that at times they are capable of rejuvenation. Are these the bodies one sees floating round in wet preparations, which are sometimes larger and sometimes smaller than a pus cell, but which are neither pus, blood, nor epithelial cells. They are not the yeast forms of monilia with which I am quite familiar. These encysted forms seem to occur mainly in patients who are receiving treatment for their vaginitis, especially when it is of the resistant type. They occur also in cultures which require subculturing. Trussell (1947) writes :

One also sees the motionless, rounded, hyaline forms with no apparent flagella. These are designated as pseudocysts which ultimately disappear and cannot be subcultured

Is it not possible that this highly-organized protozoan, and such the Trichomonas vaginalis definitely is, reacts very differently in the body and in the test tube?

We really know very little about the life cycle of Trichomonas vaginalis. I suggest that there is considerable scope here and also in the field of treatment for organized research.

\section{REFERENCES}

Andrews, M. N. (1929). J. trop. Med., 32, 237.

Balkow, N. (1935). Munch. med. Wnschr., 82, 331.

Barlow, N. (1916). New Orleans Med. Surg. J., 69, 299.

Bensen, W. (1910). Arch. Protistenk., 18, 115.

Berstine, J. B., and Rakoff, A. E. (1953). "Vaginal Infections, Infestations and Discharges", p. 256. Blakiston, New York.

Burke, E. T. (1940). " Venereal Diseases", p. $17 . \quad$ Lewis, London.

Dobell, C. (1934). Parasitology, 26, 531.

Donald, I. (1952). Brit. med. J., 2, 1223.

Donné, A. (1836), C. R. Acad. Sci. (Paris), 3, 385.

Feinberg, J. G. (1953). Nature (Lond.), 171, 1165.

Feo, L. G. (1944). Amer. J. trop. Med., 24, 195.

Fukushima, K. (1934). Nippon Fujinkagakkai Zassi, Tokyo, 29, 36.

Greenblatt, R. B., and Barfield, W. E. (1951). Amer. J. Obstet. Gynec., 62, 423.

Hantke, H. (1921). "Befunde und klinische Bedeutung der Trichomonas vaginalis." Inaugural dissertation, Breslau Kattowitz. Hegner, R. W. (1925). Amer. J. Hyg., 5, 302.

Hesseltine, H. C. (1933). Amer. J. Obstet. Gynec., 26, 46.

Hogue, M. J. (1944). Amer. J. trop. Med., 24, 255

Hosoya, S. (1954). Reported in Brit. med. J., 1, 756

Johnson, G. (1942). J. Parasit., 28, 369.

Karnaky, K. J. (1934). Urol. cutan. Rev., 38, 174.

King, A. J., Mascall, W. N., and Price, I. N. O. (1936). Lancet. 2, 18. Lanceley, F. (1954). British Journal of Venereal Diseases, 30, 163.

Liston, W. G., and Liston, W. A. (1939). J. Obstet. Gynaec. Brit. Emp., 46, 474

Lydon, F. L. (1945), Brit, med. J., 2, 384.

Lynch, K. M. (1922). J. Amer. med. Ass., 79, 1130.

McEntegart, M. G. (1954). British Journal of Venereal Diseases, 30, 167.

Schmid, A. L., and Kamniker, H. (1926). Arch. Gynäk., 127, 362.

Siebenthal, R. de (1945). Rev. med. Suisse rom., 65, 291.

Stabler, R. M., and Feo, L. G. (1942). Amer. J. trop. Med., 22, 639.

Swift, B. H. (1937). Med. J. Aust., 1, 123.

Trussell, R. E. (1947). "Trichomonas Vaginalis and Trichomoniasis." Blackwell, Oxford.

-C, and Plass, E. D. (1940). Amer. J. Obstet. Gynec., 40, 883.

Wagner, O., and Hees, E. (1935). Z Zbl. Bakt., Abt. I, 135, 310.

Wenrich, D. H. (1944). Amer. J. trop. Med., 24, 39.

Wenyon, C. M. (1926). Protozoology, 1, 660.

Whittington, M. J. (1951a). J. Obstet. Gynaec. Brit. Emp., 58, 615. - (1951b). J. Hyg. (Lond.), 49, 400.

Zinser, H. K. (1941). Geburts. u. Frauenheilk., 3, 188. 